\title{
Metatarsal Neck Fractures Are not Always Isolated Injuries: Case Report of a Lisfranc Injury
}

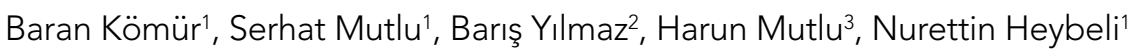 \\ ${ }^{1}$ Clinic of Orthopedics and Traumatology, Kanuni Sultan Süleyman Training and Research Hospital, İstanbul, Turkey \\ ${ }^{2}$ Clinic of Orthopedics and Traumatology, Fatih Sultan Mehmet Training and Research Hospital, İstanbul, Turkey \\ ${ }^{3} \mathrm{Clinic}$ of Orthopedics and Traumatology, Gaziosmanpaşa Taksim Training and Research Hospital, İstanbul, Turkey
}

\begin{abstract}
A 40-year-old male presented to the emergency room with tenderness, swelling, and ecchymosis in the middle/medial and dorsal aspects of his right foot after falling from a height of about 1 meter at work. He had a medial cuneiform bone fracture-dislocation, first metatarsal base luxation, displaced second metatarsal neck fracture, and nondisplaced third metatarsal neck fracture. The Lisfranc fracture-dislocation was treated with two cannulated screws using a dorsomedial approach. The displaced second metatarsal fracture was treated by open reduction and internal fixation using a Kirschner wire. The nondisplaced third metatarsal fracture was treated conservatively. (JAREM 2015; 5: 31-3)
\end{abstract}

Keywords: Luxation, Lisfranc injury, metatarsal neck fracture, medial cuneiform fracture

\section{INTRODUCTION}

Tarsometatarsal injuries that lead to separation between the midand forefoot are called Lisfranc injuries, named after the French surgeon Jacques Lisfranc de St. Martin who described tarsometatarsal amputations in the Napoleonic Era (1). A Lisfranc injury is a subtle problem that can easily escape notice. Almost half of all tarsometatarsal joint injuries are diagnosed and treated late because they are rare and have various forms. Although the injuries to these joints are usually ligamentous, they can involve bone tissue. The injuries that involve bone tissue are called fracture-dislocations and they usually result from axial and rotational forces in high-energy trauma, such as falls from height and motorcycle accidents (2). Their incidence is 1 per 60,000 annually (3). This paper reports a Lisfranc injury accompanying metatarsal neck fractures. In Lisfranc injuries occurring with metatarsal base fractures, co-occurring metatarsal neck fractures have never been reported.

\section{CASE PRESENTATION}

A 40-year-old male presented to the emergency room after injuring his right foot at work after falling $1 \mathrm{~m}$ on a plantar flexed foot. His vital signs were stable and he had an isolated injury of his right foot. The physical examination revealed pain, swelling, and ecchymosis at the proximal metatarsophalangeal joint, with tenderness and swelling broadly on the dorsal aspect of the foot and locally over the second and third metatarsals. The neurovascular examination showed no open wounds and no distal neurovascular pathology. The radiological evaluation showed second and third metatarsal neck fractures (Figure 1). Comparative radiography and computed tomography (CT) showed a nondisplaced medial cuneiform fracture and Lisfranc injury of the proximal metatarsophalangeal joint (Figure 2). A short-leg cast was applied because of the swelling, and the patient was admitted for elevation and ice application. To reduce the chance of compartment syndrome developing, the surgery was delayed for 2 days, until the edema had regressed. At surgery, the periosteum on the cuneiform bone was scraped off via a dorsomedial approach. The cuneiform fracture and first metatarsal dislocation were reduced. Using a Kirschner wire (K-wire), two $4.0 \mathrm{~mm}$ cannulated screws were inserted via fluoroscopic guidance at a $45^{\circ}$ angle through the medial cuneiform fragments, fixing the Lisfranc dislocation. The second metatarsal fracture was approached via a dorsal incision in the web between the second and third metatarsals. The extensor tendon was dissected and the fracture was reduced with a K-wire (Figure 3). The third metatarsal was not fixed because it appeared stable. A shortleg plaster was applied after checking the reduction using fluoroscopy. Three weeks later, the K-wires were removed after observing metatarsal bone union. Once union was seen radiographically, the patient was allowed gradually increasing weight-bearing. After the sixth week, the plaster cast was removed to let the foot contact the ground for full weight-bearing with full range of motion. The patient returned to work and at the 1-year follow-up his daily life activities were as they were before the injury.

\section{DISCUSSION}

The Lisfranc joint complex comprises three columns in the foot: the medial column is formed by the first metatarsal and medial cuneiform; the central column is formed by the second and third metatarsals and the intermediate and lateral cuneiforms; and the lateral column consists of the cuboid and its relationship with the fourth and fifth metatarsals. In the coronal plain, the transverse arc of the midfoot is stable. Although many ligaments contribute to this stability, the most important contribution is that of the Lisfranc ligament between the medial cuneiform and second metatarsal; this is the strongest of these ligaments (4).

Diagnosis of a Lisfranc injury begins with the patient's history and physical examination. The time of the injury and time elapsed should be determined carefully to choose among treatment alternatives. A Lisfranc injury should be considered when plantar ecchymosis is seen. The patient should be monitored closely for compartment syndrome. A radiological and CT (if necessary) study must be performed (5).

Received: $\begin{array}{r}16.02 .2014 \\ \text { Available Online Date: } 17.06 .2014 \\ \text { (c) Copyright } 2015 \text { by Gaziosmanpaşa Taksim Training and Research Hospital. Available on-line at www.jarem.org }\end{array}$
DOl: 10.5152/jarem.2014.483



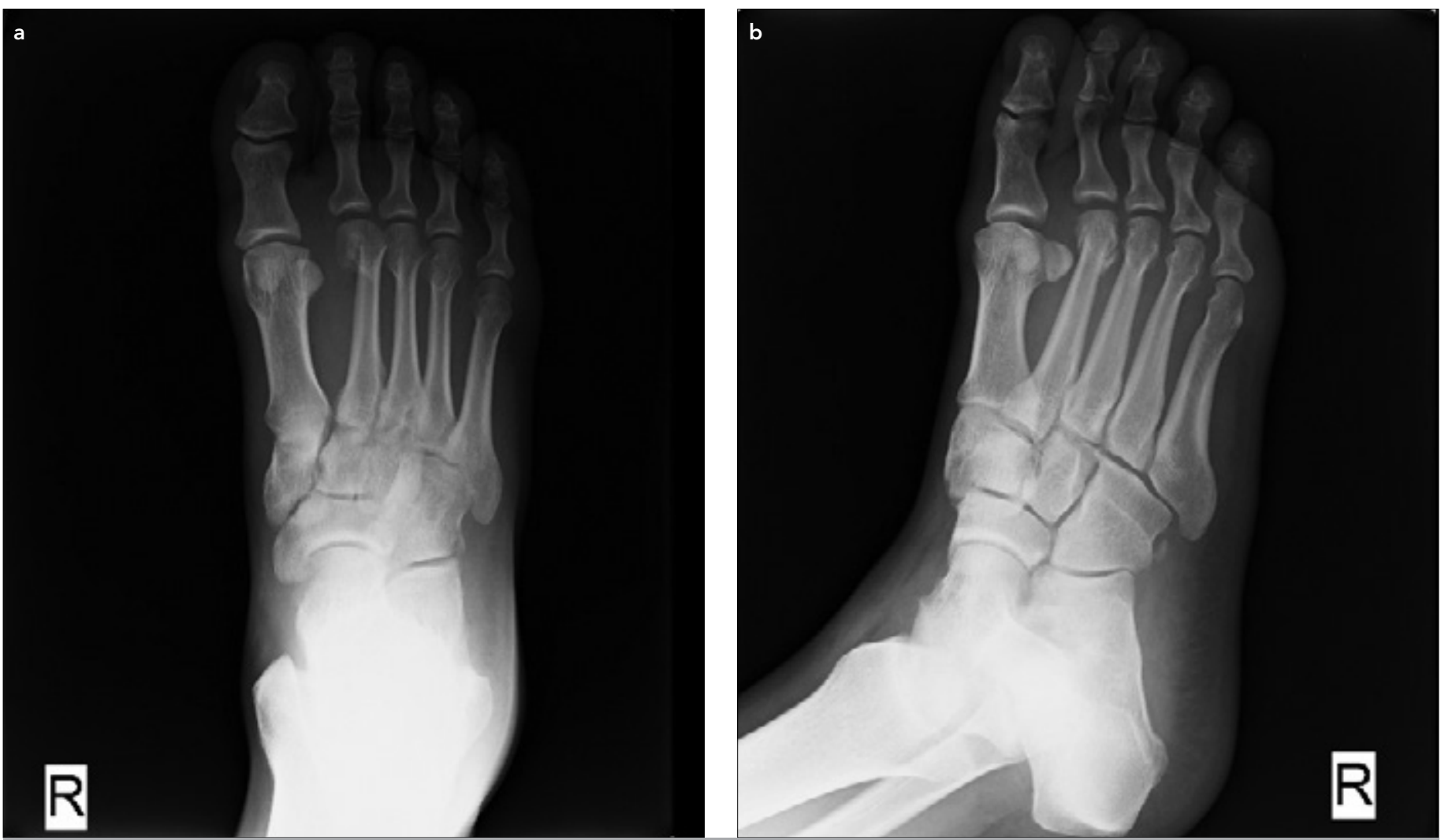

Figure 1. a, b. (a) Anteroposterior (AP) and (b) oblique X-rays showing the Lisfranc injury and metatarsal neck fracture

Lisfranc injuries usually result from one of three mechanisms: lateral translocation of a metatarsal caused by forced abduction of the forefoot; axial weight-bearing when the toes are dorsiflexed and ankle is in the equinus position; and crush injury trauma involving plantar translocation caused by force acting on the dorsal metatarsal (6). Our patient described a fall from height with the toes dorsiflexed and ankle plantar flexed (the second type described above). A fracture dislocation of the base of the first metatarsal usually occurs after such trauma. In this case, there was a fracture dislocation between the first metatarsal and medial cuneiform and neck fractures of the second and third metatarsals. In our case, the metatarsal neck fractures were probably caused by redirection of the axial force due to falling by the lifts in the front of his work shoes, which could have resulted in a rotational axial force to the forefoot and metatarsals.

Plaster casting for 6 12 weeks is recommended for non-displaced Lisfranc injuries. Surgery should be considered for displaced injuries, because of the chance of developing post-traumatic midfoot arthrosis or progressive dislocation and displacement. Closed reduction may be used for displaced dislocations and a K-wire may be used when this fails. The presence of bone fragments and entrapped soft tissues might prevent the reduction (7). Surgery should wait until any edema regresses, to reduce the risk of developing compartment syndrome. In our case, we delayed surgery for 2 days.

There are various surgical treatments for Lisfranc injuries. The current treatment is to reduce the medial and central column injuries using K-wires and to ensure osteosynthesis using cannulated screws $(2,4,7)$. It is crucial to restore the Lisfranc ligament between the middle cuneiform and second metatarsal. When the lateral column is injured, fixation of the fourth and fifth metatar-

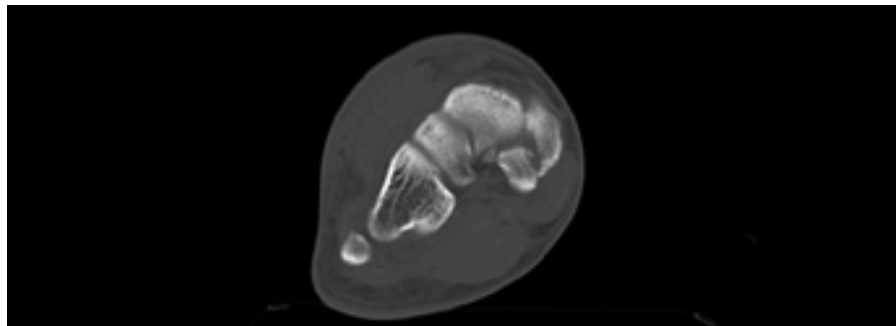

Figure 2. Computed tomography of the medial cuneiform fracture

sals to the cuboid bone is advised (8). In our case, the Lisfranc injury was reduced using K-wires and osteosynthesis was promoted using two cannulated screws. The second metatarsal fracture was also fixed using a K-wire. In some Lisfranc injuries, the metatarsal joint is luxated both proximally and distally, producing a "swimming metatarsal" (6). A Lisfranc injury accompanied by multiple metatarsal neck fracture-dislocations has also been reported (9). However, ours is the first reported Lisfranc injury accompanied by metatarsal neck fractures without luxation.

\section{CONCLUSION}

After diagnosing common isolated injuries, such as metatarsal neck fractures, problems that have a high probability of going unnoticed, such as a Lisfranc injury, should be considered. No consensus has been reached on whether to fix these using screws or K-wires. In our opinion, using a compression screw better promotes full recovery.

Informed Consent: Written informed consent was obtained from the patient who participated in this case. 

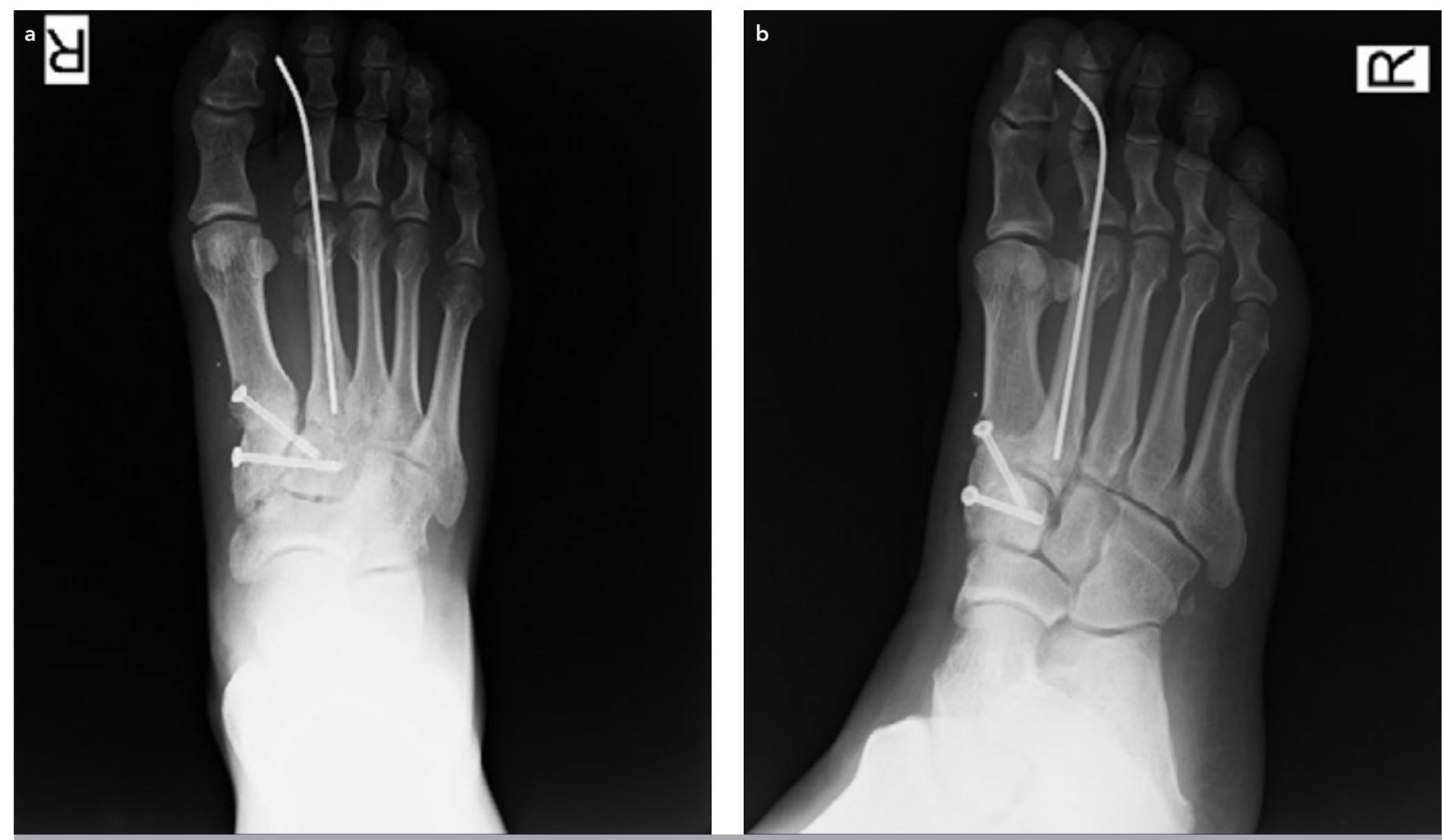

Figure 3. a, b. Fixation using two cannulated screws and a K-wire: (a) AP and (b) oblique radiographs

Peer-review: Externally peer-reviewed.

Author Contributions: Concept - B.K., N.H.; Design - H.M.; Supervision S.M., B.Y.; Resource - S.M., H.M., B.K.; Materials - N.H., B.K.; Data Collection and/or Processing - H.M., S.M.; Analysis and/or Interpretation - B.Y., N.H.; Literature Review - H.M.; Writing - B.K., N.H.; Critical Review - S.M., B.Y., N.H.

Conflict of Interest: No conflict of interest was declared by the authors.

Financial Disclosure: The authors declared that this study has received no financial support.

\section{REFERENCES}

1. Görgeç M, Esenkaya i, Nalbantoğlu U, Türkmen M. Lisfranc eklem yaralanmaları ve tedavisi. Acta Orthop Traumatol Turc 1994; 28: 240-4.

2. Scolaro J, Ahn J, Mehta S. Lisfranc fracture dislocations Clin Orthop Relat Res 2011; 469: 2078-80.

3. Hardcastle PH, Reschauer R, Kutscha-Lissberg E, Schoffmann W. Injuries to the tarsometatarsal joint: incidence, classification and treatment. J Bone Joint Surg Br 1982; 64: 349-56.
4. Miswan MFM, Singh VA, Yasin NF. Outcome of surgically treated Lisfranc injury: A review of 34 cases. Ulus Travma Acil Cerrahi Derg 2011; 17: 504-8.

5. Ross G, Cronin R, Hauzenblas J, Juliano P. Plantar ecchymosis sign: a clinical aid to diagnosis of occult Lisfranc tarsometatarsal injuries. $J$ Orthop Trauma 1996; 10: 119-22.

6. Singh PA, Singh PA, Chadha M. Lisfranc kırıklı çıkık ile birlikte ters yüzen birinci metatars ve yüzen üçüncü metatars: Nadir bir yaralanma. Acta Orthop Traumatol Turc 2010; 44: 169-71.

7. Stavlas P, Roberts CS, Xypnitos FN, Giannoudis PV. The role of reduction and internal fixation of Lisfranc fracture-dislocations: A systematic review of the literature. International Orthopaedics (SICOT) 2010; 34: 1083-91.

8. Sangeorzan BJ, Benirschke SK, Gould MT. Tarsometatarsal Lisfranc injuries: evaluation and management. In: Wiss D, ed. Master Techniques of Orthopaedic Surgery: Fractures. 2nd edition. Philadelphia, PA: Lippincott-Williams\&Wilkins, 2006: 605-17.

9. Drosos GI, Gavalas NS, Papoutsidakis AN. Concomitant Lisfranc fracture-dislocation and displaced neck fractures of the lesser metatarsals: A case report. J Foot Ankle Surg 2000; 6: 49-54. 